\title{
Engine Emissions Measurement Handbook
}




\title{
Other SAE books of interest:
}

Onboard Diagnostics and Measurement in the Automotive Industry, Shipbuilding and Aircraft Construction

By Michael Palocz-Andresen

(Product Code: R-410)

\author{
Diesel Emissions and Their Control \\ By Magdi K. Khair, W. Addy Majewski \\ (Product Code: R-303) \\ Technologies for Near-Zero-Emission \\ Gasoline-Powered Vehicles \\ By Fuquan Zhao \\ (Product Code: R-359)
}

\author{
For more information or to order a book, contact SAE International at \\ 400 Commonwealth Drive, \\ Warrendale, PA 15096-0001, USA; \\ phone 877-606-7323 (U.S. and Canada only) \\ or +1-724-776-4970 (outside U.S. and Canada); \\ fax +1-724-776-0790; \\ email CustomerService@sae.org; \\ website http://books.sae.org.
}




\title{
Engine Emissions Measurement Handbook
}

\section{HORIBA Automotive Test Systems}

\author{
Edited by \\ Masayuki Adachi and Hiroshi Nakamura
}

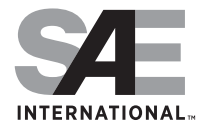

Warrendale, Pennsylvania, USA 


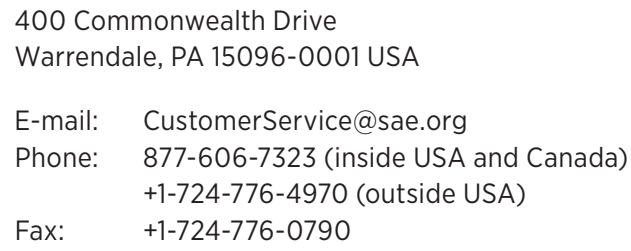

\section{Copyright $\odot 2014$ SAE International. All rights reserved.}

No part of this publication may be reproduced, stored in a retrieval system, distributed, or transmitted, in any form or by any means without the prior written permission of SAE International. For permission and licensing requests, contact SAE Permissions, 400 Commonwealth Drive, Warrendale, PA 15096-0001 USA; email: copyright@sae.org; phone: 724-772-4028; fax: 724-772-9765.

ISBN 978-0-7680-8012-4

SAE Order Number JPF-HOR-002

\section{Library of Congress Cataloging-in-Publication Data}

Engine emissions measurement handbook / edited by Masayuki Adachi and Hiroshi Nakamura.

pages $\mathrm{cm}$

Includes bibliographical references.

ISBN 978-0-7680-8012-4

1. Automobiles-Pollution control devices-Testing-Handbooks, manuals, etc. 2. Automobiles-Motors-Exhaust gas-MeasurementHandbooks, manuals, etc. I. Adachi, Masayuki (Engineer), editor. II. Nakamura, Hiroshi (Engineer), editor.

TL214.P6E57 2014

$629.25^{\prime} 28-\mathrm{dc} 23$

2013021859

Information contained in this work has been obtained by SAE International from sources believed to be reliable. However, neither SAE International nor its authors guarantee the accuracy or completeness of any information published herein and neither SAE International nor its authors shall be responsible for any errors, omissions, or damages arising out of use of this information. This work is published with the understanding that SAE International and its authors are supplying information, but are not attempting to render engineering or other professional services. If such services are required, the assistance of an appropriate professional should be sought.

\section{To purchase bulk quantities, please contact:}

SAE Customer Service

Email: CustomerService@sae.org

Phone: $\quad 877-606-7323$ (inside USA and Canada)

+1-724-776-4970 (outside USA)

Fax: $\quad+1-724-776-0790$

Visit the SAE International Bookstore at 


\section{Contents}

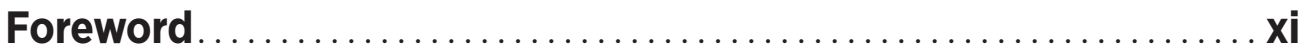

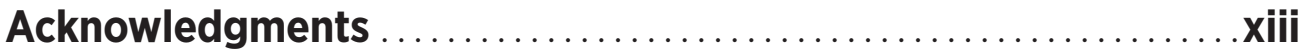

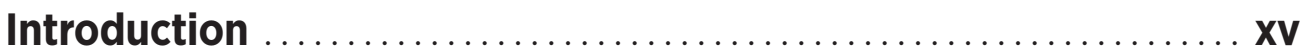

Chapter 1 Engine Emissions $\ldots \ldots \ldots \ldots \ldots \ldots \ldots \ldots \ldots \ldots \ldots$

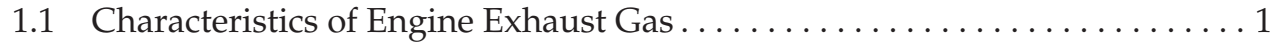

1.1.1 Major Components of Engine Exhaust Gas . . . . . . . . . . 1

1.1.2 Units Used for Exhaust Gas Components. . . . . . . . . . . . . . 1

1.1.3 Concentration Variations of Exhaust Gas Components . . . . . . . . 2

1.1.4 Exhaust Gas as Fluid (Physical Characteristics) . ........... 2

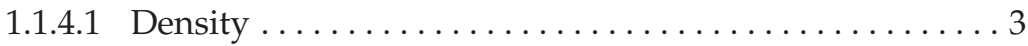

1.1.4.2 Viscosity (Coefficient of Viscosity) .............. 3

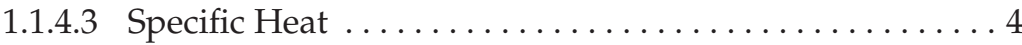

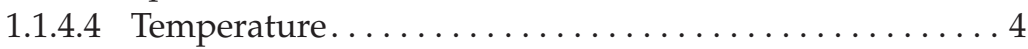

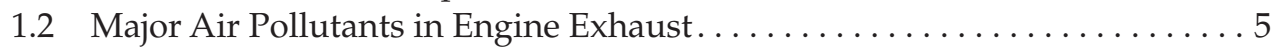

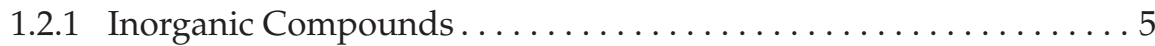

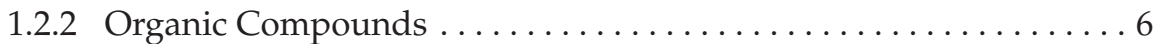

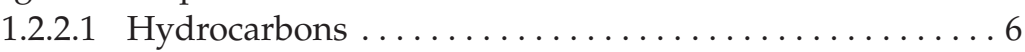

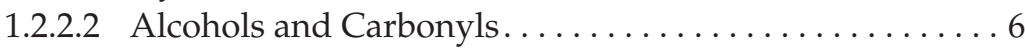

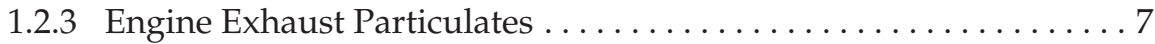

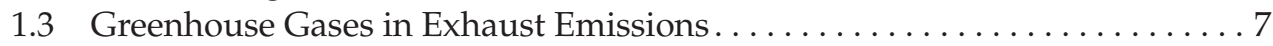

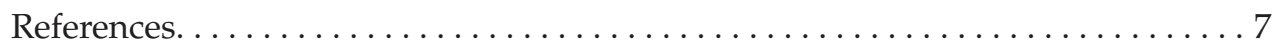

Appendix A.1 Calculations of Engine Exhaust Gas Composition . . . . . . . . . 9

A.1.1 Combustion Reaction Formula . ....................... 9

A.1.2 Calculations of Gas Component Concentration . . . . . . . . . . . . 11

A.1.3 Excess Air Ratio and Gas Component Concentration ............. 12

Chapter 2 Gaseous Emissions Measurement ................13

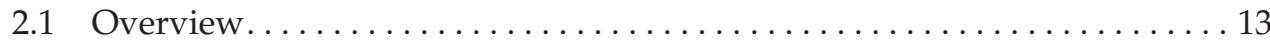

2.1.1 Direct Sampling Method . . . . . . . . . . . . . . . 13

2.1.1.1 Gas Sampling with the Direct Sampling Method. . . . . . 14

2.1.1.2 Equations of Mass Emission ............... 14

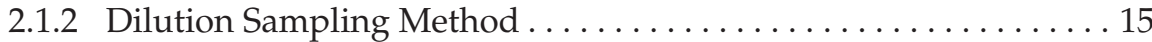

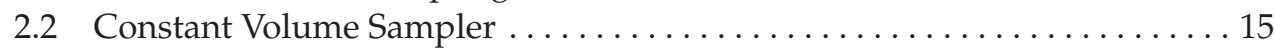

2.2.1 Overview of the Constant Volume Sampler............. 15

2.2 .2 System Structure . . . . . . . . . . . . . . . . . . . 16

2.2.2.1 Mixing Tee $\ldots \ldots \ldots \ldots \ldots \ldots \ldots \ldots \ldots \ldots \ldots \ldots \ldots \ldots \ldots \ldots \ldots$ 


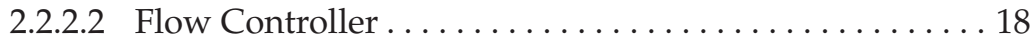

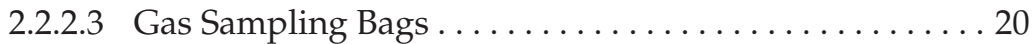

2.2 .3 Calculation of Mass Emission. . . . . . . . . . . . . . . 21

2.2.4 System Setup and System Check . . . . . . . . . . . . . 22

2.2.4.1 Setting the Dilution Flow Rate ............. 22

2.2.4.2 Calibration of the Dilution Flow Rate ........... 24

2.2.4.3 Total System Verification ................ 26

2.3 Measurement of Ultra-Low-Emission Vehicles

by the CVS Method . . . . . . . . . . . . . . . . . 27

2.3.1 Compensation of Background (Dilution Air) . . . . . . . . . . 29

2.3.1.1 Active Carbon Filter . . . . . . . . . . . . . . . . . . . 29

2.3.1.2 Dilution Air Refiner .................... 29

2.3.1.3 Proportional Ambient Sampling Method . . . . . . . . . . 30

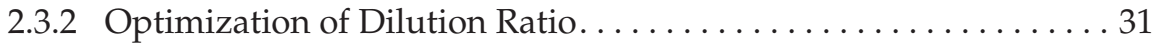

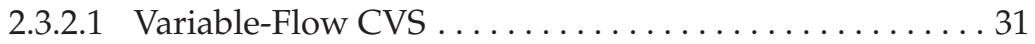

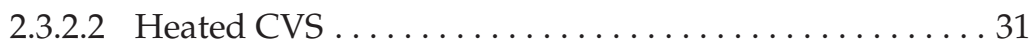

2.3.2.3 Dehumidification of the Dilution Air. . . . . . . . . 32

2.3 .3 Preventing HC Hang-Up. . . . . . . . . . . . . . . . . . 32

2.3.3.1 Selection of Materials . . . . . . . . . . . . . 32

2.3.3.2 Separate Sample Lines for Low-Concentration

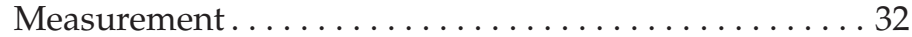

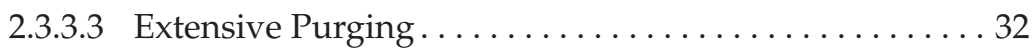

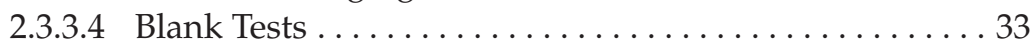

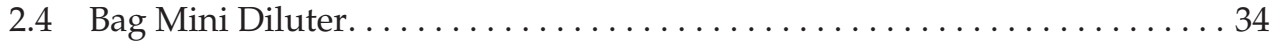

2.4.1 System Configuration and Features................ 34

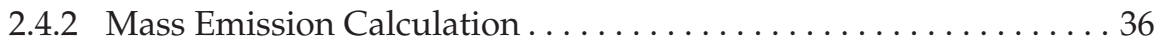

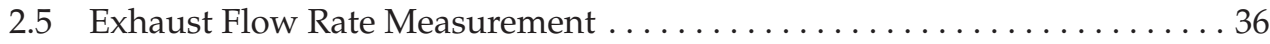

2.5.1 Summary of Exhaust Flow Measurement. . . . . . . . . . . 36

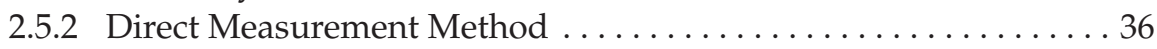

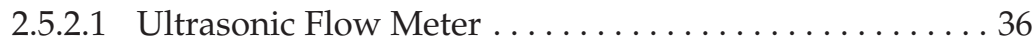

2.5.2.2 Pitot Tube Flow Meter . . . . . . . . . . . . . . . . . 39

2.5 .3 Indirect Measurement Method . . . . . . . . . . . . . . . 40

2.5.3.1 Intake Air Flow and Fuel Flow . . . . . . . . . . 40

2.5.3.2 Intake Air Flow and Air-to-Fuel Ratio . . . . . . . . . . 41

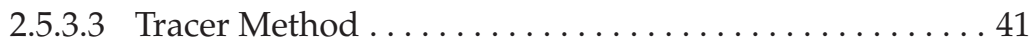

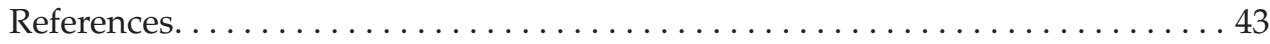

\section{Chapter 3 Measurement of Particle Emissions and}

Black Smoke .......................................... 45

3.1 PM Mass Filter Weighing (Balance) Method .................. 47

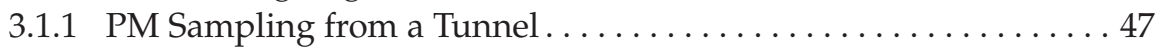

3.1.1.1 Full-Flow Dilution Tunnel ............... 47

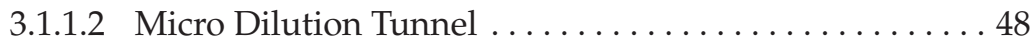

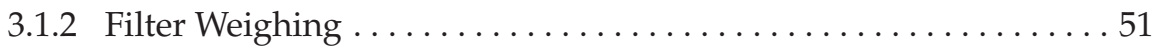


3.1.3 Separate Analysis of Soluble Organic Fraction . . . . . . . . . . . 53

3.2 Other Methods for Exhaust Particle Mass Measurement . . . . . . . . . . . 53

3.2.1 Filter Combustion Method for Measuring Low-Mass PM. . . . . . . 53

3.2.2 Continuous Measurement of PM Mass Concentration. . . . . . . . . 55

3.3 Particle Number Measurement . . . . . . . . . . . . . . . . . . . . . . 56

3.3.1 Continuous Measurement of Solid Particle Number . . . . . . . . . . 56

3.3.2 Particle Size Distribution Measurement .............. 57

3.4 Measurement of Black Smoke . . . . . . . . . . . . . . . . . . . . . 60

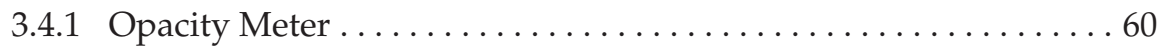

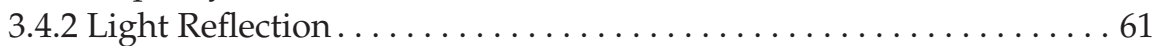

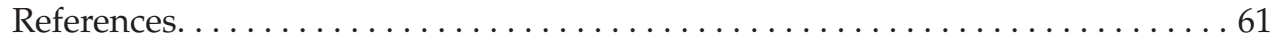

\section{Chapter 4 Evaporative Emissions Measurement ............6 63}

4.1 Outline of Evaporative Emission Regulation $\ldots \ldots \ldots \ldots \ldots \ldots \ldots \ldots$

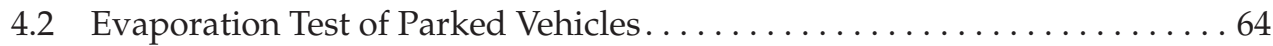

4.2 .1 Outline of the Test. . . . . . . . . . . . . . . . . . . 64

4.2.1.1 Variable Pressure Absorption System . . . . . . . . . 66

4.2.1.2 Calibration and HC Retention Test. . . . . . . . . . . . 66

4.2.2 Analyzer for Evaporative Emission Measurement . . . . . . . . . 67

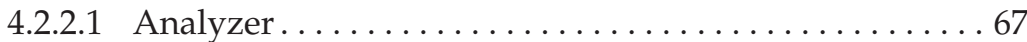

4.2.2.2 Calculation of Evaporation Rate............667 67

4.3 Running Loss Evaporation Test. . . . . . . . . . . . . . . . . . 67

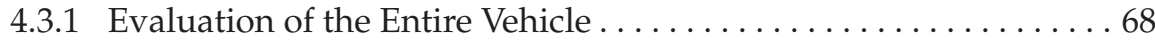

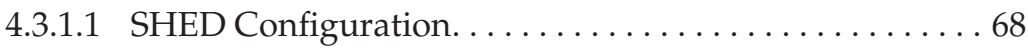

4.3.1.2 Fuel Temperature Profile ................ 69

4.3.2 Evaluation of Evaporative Emission by the

Point-Source Method ........................ 70

4.4 Evaporative Test After Vehicle Driving $\ldots \ldots \ldots \ldots \ldots \ldots \ldots \ldots \ldots \ldots \ldots \ldots \ldots \ldots \ldots$

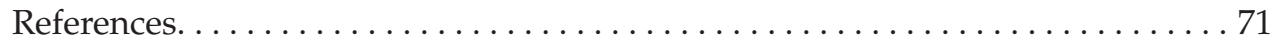

Chapter 5 Principles of Exhaust Gas Analyzers.............. 73

5.1 Analytical Principles for Regulated Emission Components of Interest. . . . 73

5.2 Non-Dispersive Infrared Detection. . . . . . . . . . . . . . . . 74

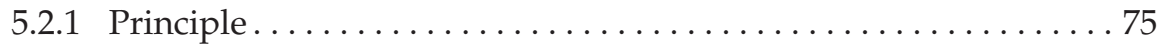

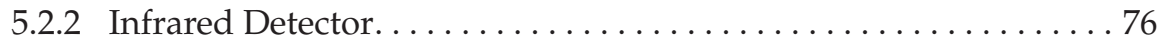

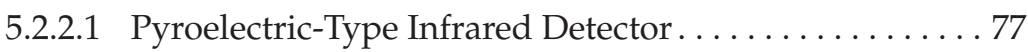

5.2.2.2 Pneumatic Cell-Type Detector. . . . . . . . . . . . 78

5.2.2.3 Detector Using Lead Selenide................... 79

5.2.3 Application to Exhaust Gas Analyzers . . . . . . . . . . . . 80

5.2.3.1 Interference Compensation by AS Method ........ 80

5.2.3.2 Adjustment of Water Concentration in

Calibration Gas . . . . . . . . . . . . . . . . . . . . . 81

5.3 Flame Ionization Detection. . . . . . . . . . . . . . . . . 82

5.3.1 Principle of Flame Ionization Detection $\ldots \ldots \ldots \ldots \ldots \ldots$ 
5.3.2 Application to Exhaust Gas Analyzer ................. 83

5.3.2.1 Separate Measurement of Methane . . . . . . . . 83

5.3.2.2 Compensation of Oxygen Interference $\ldots \ldots \ldots \ldots \ldots 84$

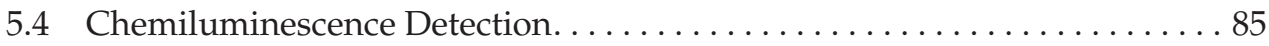

5.4.1 Principle of Chemiluminescence Detection . . . . . . . . . . 86

5.4.2 Application to the Exhaust Gas Analyzer. . . . . . . . . . . . . 87

5.4.2.1 Atmospheric Pressure-Type and Vacuum

Pressure-Type NOx Analyzers . . . . . . . . . . . . 8 87

5.4.2.2 Options of Sample Dehumidification .......... 88

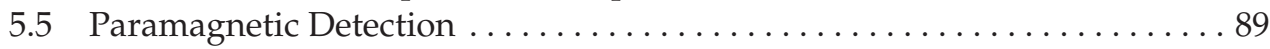

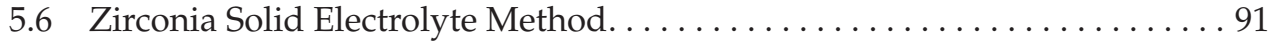

5.6.1 Zirconia-Type Air-to-Fuel Ratio Sensor. . . . . . . . . . . . . 91

5.6.2 Zirconia-Type Nitrogen Oxide Sensor. . . . . . . . . . . . . . . 92

5.7 Fourier Transform Infrared Spectroscopy . . . . . . . . . . . . . . 93

5.7.1 Principle of Fourier Transform Infrared Gas Analyzer. . . . . . . . . . . . 93

5.7.2 Analyzer Configuration and Target Gas Components . . . . . . . . 94

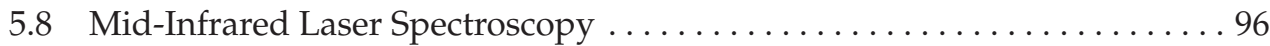

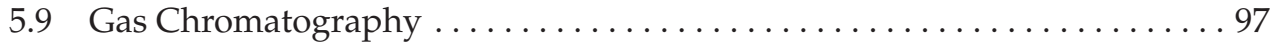

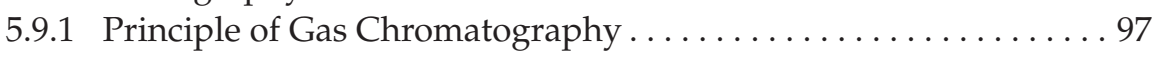

5.9.2 System Components and Conditions ............... 98

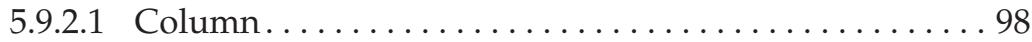

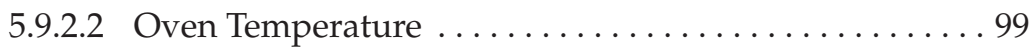

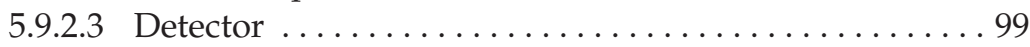

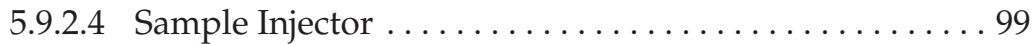

References......................................... 100

\section{Chapter 6 Testing Equipment for Vehicle Emissions............101}

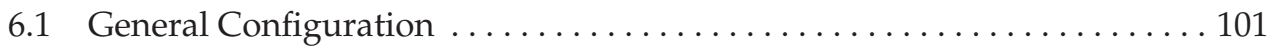

6.2 Equipment for Simulating Actual Driving Conditions . . . . . . . . . . 103

6.3 Equipment for Gas Component Analysis. . . . . . . . . . . . . . . 103

6.3.1 Constant Volume Sampling System. . . . . . . . . . . . . . . . . 103

6.3 .2 Exhaust Gas Analyzer . . . . . . . . . . . . . . . . . . . . . . . 104

6.4 Particle Measurement Equipment. . . . . . . . . . . . . . . . . 105

6.5 Driving Aid Equipment/Automatic

Driving Equipment . . . . . . . . . . . . . . . . . . . 105

6.5 .1 Driver's Aid System . . . . . . . . . . . . . . . . . . . . . 105

6.5 .2 Robot Driver . . . . . . . . . . . . . . . . . . 105

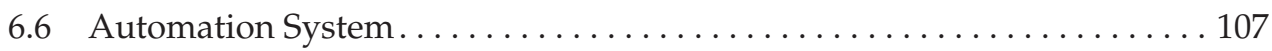

6.6.1 Laboratory Automation System . . . . . . . . . . . . . . . . 107

6.6.2 Full-Automatic Unmanned Testing System . . . . . . . . . . . . 108

Chapter 7 Practical Measurement of Engine Emissions......... 111

7.1 Emission Measurements for Powertrain Development............. 111

7.1.1 Air-to-Fuel Ratio and Excess Air Ratio . . . . . . . . . . . . 111 
7.1.1.1 Calculating from Exhaust Gas Concentrations. . . . . . . 111

7.1.1.2 Real-Time Measurement Using Zirconia Sensor . . . . . . 114

7.1.2 Exhaust Gas Recirculation Ratio . . . . . . . . . . . . . . 115

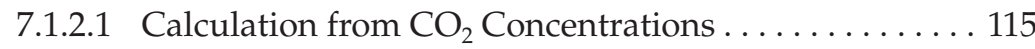

7.1.2.2 Transient Measurement of EGR Ratio . . . . . . . . . . 116

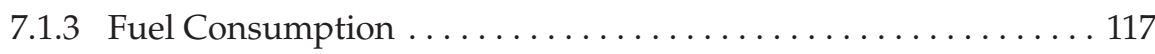

7.1.3.1 Vehicles Using Combustion Engine for Power Source . . . . . . . . . . . . . . . . . . . 117

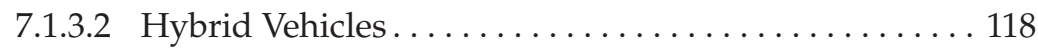

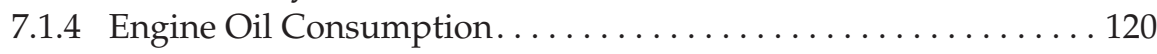

7.1.4.1 S-trace Method........................ 121

7.1.4.2 Calculation of Oil Consumption............. 122

7.1.5 Catalytic Converter Evaluation . . . . . . . . . . . . . 124

7.1.5.1 Three-Way Catalytic Converter Evaluation .... . . . . . . 124

7.1.5.2 Evaluation of Aftertreatment System for Nitrogen

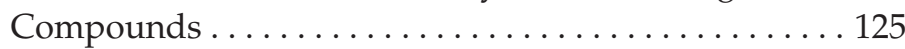

7.1.5.3 Evaluation of Diesel Particulate Filter . . . . . . . . 128

7.1.5.4 Evaluation of Aftertreatment Catalyst Using

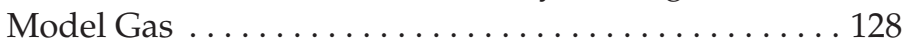

7.1.6 Measurement of Oxygenated Hydrocarbons . . . . . . . . . . . . . 129

7.1.6.1 Quantitative Estimation of Alcohols . . . . . . . . . . 129

7.1.6.2 Quantitative Estimation of Carbonyl Compounds .... 131

7.1.6.3 Measurement of Non-Methane Organic Gases . . . . . . 133

7.1.7 Measurement of Greenhouse Gases. . . . . . . . . . . . . . . . 136

7.1 .8 Modal Mass Analysis. . . . . . . . . . . . . . . . . . . . 137

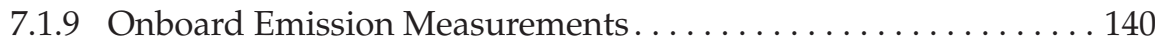

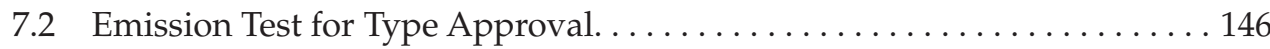

7.2.1 Scope of Regulation and Test Procedures . . . . . . . . . 146

7.2.2 Common Test Procedure ..................... 147

7.2.2.1 Test Cycles ......................... 148

7.2.2.2 Off-Cycle Emission Tests . . . . . . . . . . . . . . . . 149

7.3 Emission Test for Production Vehicles . . . . . . . . . . . . . . . 151

7.3.1 Random Sampling Inspection on Production Line . . . . . . . . . 151

7.3.2 In-Use Vehicle Measurement . . . . . . . . . . . . . . . . . . . . . . 151

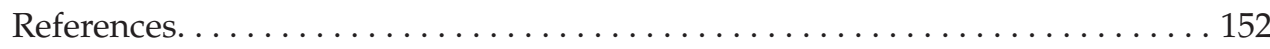

\section{Chapter 8 Emission Regulations Around the World and} Worldwide Harmonization of Technical Regulations .......... 157

8.1 Emission Regulations Around the World. . . . . . . . . . . . . . . 157

8.1 .1 Japanese Emission Standards . . . . . . . . . . . . . . . 157

8.1.1.1 History and Regulatory Process .............. 157

8.1.1.2 Recent Trend ....................... 159

8.1 .2 United States Emission Standards . . . . . . . . . . . . . . . . . 160

8.1.2.1 History and Regulatory Process ............... 160 
8.1.2.2 Recent Trend ......................... 161

8.1 .3 European Union Emission Standards . . . . . . . . . . . . . . 163

8.1.3.1 History and Regulatory Process ............. 163

8.1.3.2 Recent Trend ......................... 164

8.1 .4 Other Nations and Regions. . . . . . . . . . . . . . . . . . . 164

8.2 Worldwide Harmonization of Technical Regulations and

Mutual Recognition of Approval. . . . . . . . . . . . . . . . . . . . 165

8.2.1 1958 Agreement and ECE Regulation . . . . . . . . . . . . . 166

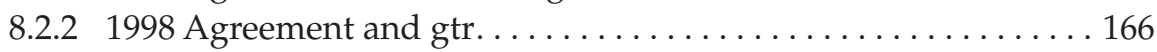

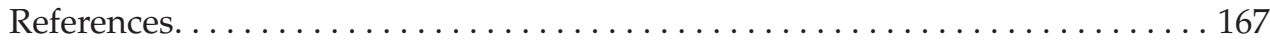

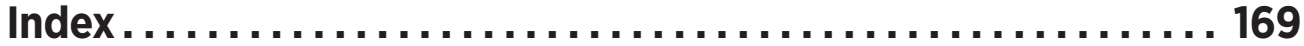




\section{Foreword}

The HORIBA Group marked its 60th anniversary in 2013, growing together with the advancement of a variety of industries in the world.

During these years, engine emission measurement technologies have made remarkable progress, which has corresponded to the growth of the automotive industry, and have become HORIBA's largest business segment. This history reflects an expansion on the concept of simple gas analyzers to more sophisticated, integrated laboratory automation systems, ranging in purpose from sample handling to test automation. The development needs of low-emission, high-efficiency engines; alternative fuels; and catalyst systems have provided HORIBA with great opportunities to develop new technologies. We deeply appreciate the valuable input and constructive relationship that has been developed between our company, the industry, and our valued customers.

Recent advancements in computers and information technology have somewhat overshadowed the intrinsic analytical technologies and their historical background. This handbook summarizes the necessary knowledge of analytical methods and advanced applications for the latest engine emission measurements.

I hope this handbook will help with powertrain R\&D activities, and further contribute to the growth of the automotive industry.

May 2013

Atsushi Horiba

Chairman, President \& CEO 


\section{Acknowledgments}

This book is the result of extensive teamwork at HORIBA. The following contributors should be recognized, as their expertise was critical in the creation of the Engine Emissions Measurement Handbook. Their effort and technical input were invaluable to the success of this project.

Masayuki Adachi, Masanobu Akita, Tetsuji Asami, Ichiro Asano, Hirokazu Fukushima, Kenji Hara, Masahiro Higuchi, Aoi Ikushima, Kaori Inoue, Yuki Itoga, Yoshinori Kato, Hiroshi Kawamura, Nobutaka Kihara, Kenji Kondo, Yoshinori Kondo, Satoshi Koyama, Tatsuki Kumagai, Takeshi Kusaka, Hiroshi Nakamura, Shigeo Nakamura, Shigeru Nakatani, Satoko Nishikawa, Masashi Nishimura, Shinji Noguchi, Masaru Miyai, Shinichi Murakami, Yoichi Okada, Yoshinori Otsuki, Masashi Sakaguchi, Tetsuo Sasaki, Katsushi Taguchi, Yasushi Takahashi, Kei Tanaka, Masatsune Tokuhira, Osamu Yamada, Masahiro Yamaoka, and Tomoshi Yoshimura (HORIBA, Ltd.).

Mike Akard, Sally Every, Neal Harvey, Tim Nevius, Scott Porter, and Rick Rooney (HORIBA Instruments Incorporated). 


\section{Introduction}

The origin of the internal combustion engine dates back to 1860 when Jean-Joseph Etienne Lenoir developed a gas engine. This was followed by Nikolaus Otto's invention in 1876 of the Otto cycle engine, which is the fundamental concept of current engines. The development of Rudolph Diesel's engine in 1893 helped accelerate the industrial revolution as the primary power source during the late 19th and early 20th centuries.

Progress in the development of the internal combustion engine has become the driving force for the development of the automobile, fulfilling the human appetite for mobility. Mass production since the early 20th century has made automobiles an affordable purchase for many in the consumer market. In the late 20th century, the automotive market expanded worldwide.

Consumption of fossil fuels, namely coal and petroleum, has rapidly expanded worldwide with the economic development and infrastructure advancements in many countries. Most of these countries have experienced health hazards related to automotive emission, such as photochemical smog, which was first identified in Los Angeles in the 1950s. The scientific community has repeatedly issued an alarm that continued consumption of huge amounts of fossil fuels will result in global warming with serious implications on the environment.

Under such circumstances, technology to improve energy efficiency and reduce emissions has become the top priority to minimize environmental impact by automobiles. The auto industry has responded to this requirement by developing advanced engines and vehicles with high efficiency and low emissions such as super clean diesel engines, hybrid electric powertrains, fuel cell vehicles, and vehicles with alternative fuels from biomass. In fact, the concentrations of some pollutants in exhaust gas from recent advanced vehicles, called Super-Ultra-LowEmission Vehicles, can be lower than those in ambient air.

In the process of establishing new energy-efficient emission-reduction technologies during the past half a century, analytical instruments for emission measurement have provided essential research and development support, and become fundamental tools. Requirements for future analytical instruments are anticipated to be even more sophisticated and diversified with the continued progress of automotive technologies. 
The year 2013 is the 60th anniversary of the foundation of the HORIBA group, and the 50th year since the launch of the first HORIBA emissions measurement system. This handbook provides a wide range of topics: from the fundamental principles of analytical technologies, to the most recent measurement applications for advanced emission reduction systems. I hope this handbook will provide useful technical support for the engineers engaged in the development of new emission technologies, a field of study in which reliable, adequate analytical instruments are indispensable.

In closing, I would like to express my great appreciation to SAE International, Mr. Toshiji Yamazaki, Mr. Neal Harvey, and all the authors who contributed their knowledge toward the publication of this handbook.

May 2013

Kozo Ishida

Executive Vice President

HORIBA, Ltd. 